\title{
MUTUAL ACTIVATION AND SORPTION ABILITY \\ OF RARE CROSS-LIN KED NETWORKS IN INTERGEL SYSTEM BASED ON POLYMETHACRYLIC ACID AND POLY-4-VINYLPYRIDINE HYDROGELS IN RELATION TO LAN THANUM IONS
}

\author{
Talkybek J umadilov ${ }^{1, *}$, Zharylkasyn Abilov², Juozas Grazulevicius ${ }^{3}$, \\ Nazym Zhunusbekova1, Ruslan Kondaurov², Laura Agibayeva², Auez Akimov
}

https://doi.org/10.23939/chcht11.02.188

\begin{abstract}
Sorption ability of intergel system polymethacrylic acid hydrogel (gPMAA) - poly-4-vinylpyridine hydrogel (gP4VP) in relation to lanthanum ions is studied. Hydrogels mutual activation in an aqueous medium, particularly dependencies of swelling coefficient, specific electric conductivity and $\mathrm{pH}$ of water solutions are studied. Maximum activation of hydrogels occurs at gPMAA:gP4VP molar ratio 3:3. Maximum total binding degree of polymer chain in the intergel system is observed at the ratio gPMAA:gP4VP $=1: 5$. It has the value of $73.13 \%$, which is much higher than at initial hydrogels: gPMAA binding degree is $55.17 \%$, gP4VP $55.00 \%$. The obtained results point to the fact that at this ratio there are significant changes of electrochemical, conformational and sorption degrees of initial macromolecules in intergel system.
\end{abstract}

Keywords: intergel systems, polymethacrylic acid, poly4-vinylpyridine, remote interaction, $\mathrm{La}^{3+}$ ions, sorption, desorption.

\section{Introduction}

Previous studies were devoted to investigation of hydrogels remote interaction effect [1-6]. In the result of these studies it was found that hydrogels remote interaction provides significant changes of their electrochemical and conformational properties. Further investigations were devoted to the study of influence of

\footnotetext{
${ }^{1}$ JSC "Institute of chemical sciences after A.B. Bekturov" 106, Sh. Valikhanov St., 050001 Almaty, Republic of Kazakhstan

${ }^{2}$ Al-Farabi Kazakh National University,

71, Al-Farabi Ave., 050001 Almaty, Republic of Kazakhstan

${ }^{3}$ Kaunas University of Technology,

73, K. Donelaicio St., 44029 Kaunas, Lithuania

jumadilov@mail.ru

(C) Jumadilov T., Abilov Z., Grazulevicius J., Zhunusbekova N., Kondaurov R., Agibayeva L., Akimov A., 2017
}

different factors on polymer hydrogels remote interaction in intergel systems [7-11].

In present technologies of concentration and recovery of rare earth and other elements in hydrometallurgy are based on the use of ion exchange resins. However, ion exchangers do not have high degree of selective recovery of metals and their regeneration is a complex process. Moreover, application of ion exchange resins is directed to selective recovery of one metal whereas industrial solutions generally contain several valuable components. In this regard the aim of this work is study of sorption ability of intergel system based on hydrogels of polymethacrylic acid and poly-4vinylpyridine in relation to lanthanum ions.

\section{Experimental}

Conductometer "MARK-603" (Russia) was used for measurement of specific electric conductivity and hydrogen ions concentration was measured on $\mathrm{pH}$-meter Metrohm $827 \mathrm{pH}$-Lab (Switzerland). Mass of samples was estimated on analytical scales SHIMADZU AY220 (Japan). $\mathrm{La}^{3+}$ ions concentration was determined on spectrophotometers Perkin Elmer Lambda 35 (USA) and SF-46 (Russia).

\subsection{M aterials}

Studies were carried out in an aqueous medium and in $0.005 \mathrm{M}$ solution of 6 -water lanthanum nitrate. Hydrogels of polymethacrylic acid (gPMAA) were synthesized in the presence of crosslinking agent $\mathrm{N}, \mathrm{N}$ methylene-bis-acrylamide and redox system $\mathrm{K}_{2} \mathrm{~S}_{2} \mathrm{O}_{8}-$ $\mathrm{Na}_{2} \mathrm{~S}_{2} \mathrm{O}_{3}$ in an aqueous medium. Synthesized hydrogels were crushed into small dispersions and continuously washed with distilled water until a constant conductivity value of aqueous solutions appeared. Poly-4-vinylpyridine hydrogel (gP4VP, synthesized by Sigma-Aldrich) crosslinked with divinylbenzene was used. 
For investigation task from synthesized hydrogels intergel pairs gPMAA-gP4VP were created. Swelling coefficients of hydrogels are: $K_{s w}($ gPMAA $)=20.65 \mathrm{~g} / \mathrm{g}$, $K_{s w}(\mathrm{gP} 4 \mathrm{VP})=3.27 \mathrm{~g} / \mathrm{g}$. With increase of gP4VP share in intergel system gPMAA concentration decreased from 5.97 to $0.98 \mathrm{mmol} / \mathrm{l}$ (interval of gPMAA:gP4VP ratios 6:0-1:5). Concentration of gP4VP with decrease of gPMAA increased from 0.99 to $5.99 \mathrm{mmol} / \mathrm{l}$ (interval of gPMAA:gP4VP ratios 1:5-0:6).

\subsection{Electrochemical investigations}

Experiments were carried out at room temperature. Studies of intergel system were made in the following order: each hydrogel in dry initial state was put in glass filter with pores permeable to low molecular ions but nonpermeable to hydrogel dispersion. After that filters with hydrogels were put into glasses with aqueous solutions and lanthanum nitrate solutions. Electric conductivity and $\mathrm{pH}$ of solutions were measured in the presence of hydrogels.

\subsection{Determination of hydrogels swelling}

Swelling coefficient was calculated by equation:

$$
K_{s w}=\frac{m_{2}-m_{1}}{m_{1}}
$$

where $m_{1}-$ mass of dry hydrogel; $m_{2}-$ mass of swelled hydrogel.

\subsection{Lanthanum ions desorption}

After sorption of lanthanum ions each hydrogel separately from another was put on lanthanum ions desorption in $96 \%$ ethyl alcohol solution and in $2 \mathrm{M}$ nitric acid [12].

Desorption degree was calculated by the equation:

$$
R_{\text {des }}=\frac{m_{\text {des }}}{m_{\text {sorp }}} \cdot 100 \%
$$

where $m_{d e s}-$ mass of desorbed lanthanum; $m_{\text {sorp }}-$ mass of sorbed lanthanum.

\subsection{M ethodology of lanthanum ions determination}

Methodology of lanthanum ions determination is based on formation of colored complexed compound of organic analytic reagent Arsenazo(II)I with lanthanum ions [13].

Total binding degree of internode links of polymer chain was calculated according to the equation:

$$
\theta=\frac{v_{\text {sorb }}}{v} \cdot 100 \%
$$

where $v_{\text {sorb }}$ - quantity of sorbed lanthanum, mol; $v-$ quantity of polymer sample (if there are 2 hydrogels in solution, it is calculated as a sum of quantities of each of them), mol.

\section{Results and Discussion}

\subsection{Study of Mutual Activation of $H$ ydrogels gPM AA and gP4VP in Intergel System}

In the presence of intergel system in an aqueous medium various processes which impact ionic equilibrium in solution occur. Such processes are:

1. Dissociation of $-\mathrm{COOH}-$ groups of internode links:

$-\mathrm{COOH} \rightarrow \mathrm{COO}^{-\cdots} \mathrm{H}^{+} \rightarrow-\mathrm{COO}^{-}+\mathrm{H}^{+}$

It should be noted that dissociation occurs stepwise: firstly, there is ionization with ionic pairs formation, after that ionic pairs partially dissociate to separate ions.

2. Ionization and partial dissociation of nitrogen heteroatom in pyridine ring:

$$
\equiv \mathrm{N}+\mathrm{H}_{2} \mathrm{O} \rightarrow \equiv \mathrm{NH}^{+} \ldots \mathrm{OH}^{-} \rightarrow \equiv \mathrm{NH}^{+}+\mathrm{OH}^{-}
$$

3. Interaction of nitrogen heteroatom with proton, which was cleaved from carboxyl group:

$$
\equiv \mathrm{N}+\mathrm{H}^{+} \rightarrow \equiv \mathrm{NH}^{+}
$$

4. Formation of water molecules by interaction of $\mathrm{H}^{+}$and $\mathrm{OH}^{-}$ions, which were formed in the result of reaction of functional groups with water molecules:

$$
\mathrm{H}^{+}+\mathrm{OH}^{-} \rightarrow \mathrm{H}_{2} \mathrm{O}
$$

These interactions result in the state at which there are no counterions at some part of charged functional groups. Concentration of ionized groups without counterions is in dependence on initial molar ratios of polymer networks and other factors.

The appearance of high concentration of hydroxyl ions is possible in case of interaction of vinylpyridine links with water molecules in accordance with Eq. (2).

In Fig. 1 dependence of specific electric conductivity change on hydrogels molar ratios in time is presented. During remote interaction there appear areas of minimum and maximum electric conductivity leading to increase of electric conductivity of electric conductivity almost for all hydrogels molar ratios. As it can be seen from the figure minimum electric conductivity is observed at the ratio $4: 2$ for the entire time of hydrogels remote interaction. Low values of electric conductivity are also observed at the ratio gPMAA:gP4VP $=1: 5$. The area of maximum conductivity is the ratio gPMAA:P4VP $=3: 3$.

Minimum values of conductivity are due to binding of proton which was cleaved from carboxyl group by nitrogen heteroatom of vinylpyridine. When put in water medium both hydrogels begin to swell in the result of 
interaction with water molecules. Carboxyl groups are firstly ionized and after that they dissociate to carboxylate anions $-\mathrm{COO}^{-}$and hydrogen ions (protons) $\mathrm{H}^{+}$. Ionization of cationic hydrogel poly-4-vinylpyridine in an aqueous medium occurs in the result of accession of hydrogen ions formed at carboxyl groups dissociation and water molecules of ions $\mathrm{H}^{+}, \mathrm{OH}^{-}$. Hydrogels long-range effect provides decrease of positive charges in an aqueous medium.

High electric conductivity values, in turn, point to the predominance of carboxyl groups dissociation over proton association by nitrogen heteroatoms of polybasis. The main reason of this phenomenon is conformational changes of links in internode chains. At certain concentrations charged $\mathrm{NH}^{+}$groups may form intramolecular crosslinks $\geq \mathrm{N} . . . \mathrm{H}^{+} \ldots \mathrm{N} \equiv$, which provide folding of macromolecular globes and decrease of proton binding.

Fig. 2 shows dependence of $\mathrm{pH}$ of water solutions on molar ratios of gPMAA:gP4VP in time. With predominance of polyacid there is an increase of hydrogen ions concentration with time. Clear minimums are observed at the presence of only polyacid (ratio gPMAA:gP4VP = 6:0) and at the ratio gPMAA:gP4VP = $3: 3$. With increase of polybasis share $\mathrm{pH}$ of solution increases and reaches its maximum at the ratio gPMAA:gP4VP $=1: 5$. With time $\mathrm{pH}$ values of the maximum decrease, indicating additional proton release.

Comparing low hydrogen concentration and conductivity high values at the ratio gPMAA:gP4VP $=3: 3$ it may be concluded that at this ratio there is an occurrence of maximum activation of hydrogels in the intergel system.

Concentration of ions in the studied intergel system is in direct dependence on swelling rate and hydrogels

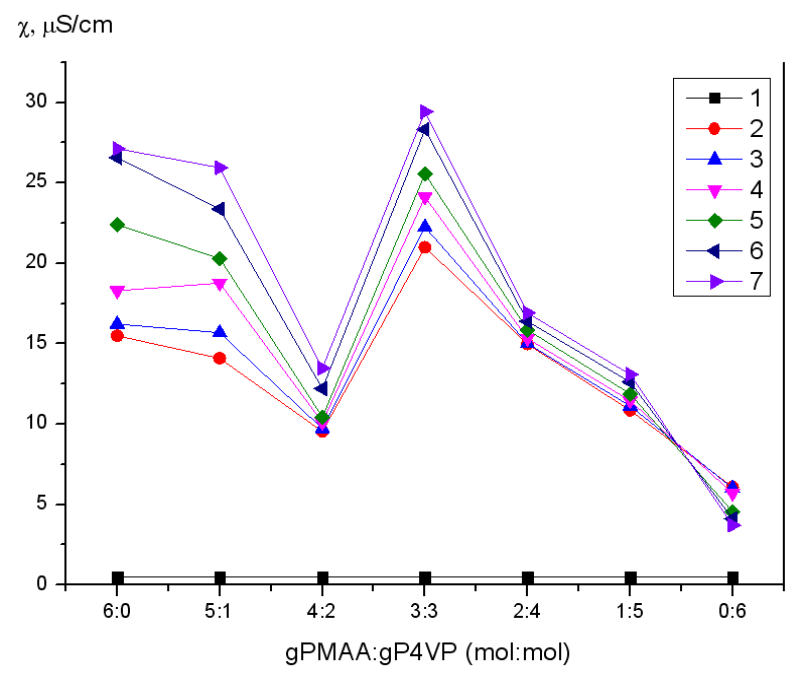

Fig. 1. Dependence of specific electric conductivity on gPMAA and gP4VP hydrogels molar ratios in time (h): 0 (1); 0.5 (2); 1 (3); 2 (4); 6 (5); 24 (6) and 48 (7) concentration in water solution. Rates of swelling and deprotonization are in dependence on the nature of hydrogel, crosslinking degree, dispersity, and longitudinal size of polymer hydrogels.

There is excess of $\mathrm{H}^{+}$ions due to high rate of swelling and dissociation of $\mathrm{COOH}$-groups and low swelling rate of basic groups and their low concentration. Increase of $\mathrm{OH}^{-}$ions in water medium is due to low swelling speed and interaction of basic functional groups with protons. It is possible in case of occurrence of the ionization and partial dissociation of nitrogen heteroatom in pyridine ring, in the result of which hydroxyl anions are released in solution. In parallel there is interaction of free proton with pyridine ring and concentration of positively charged ions in solution sharply decreases.

Dependence of acid and basic hydrogels swelling coefficients on molar ratios in time is presented in Figs. 3 and 4.

Dependence of polymethacrylic acid swelling coefficient on hydrogels molar ratios is presented in Fig. 3. As it can be seen from the figure polybasis share increase provides gPMAA swelling increase. Maximum swelling is observed at gPMAA:gP4VP $=1: 5$ ratio at $48 \mathrm{~h}$ of hydrogels remote interaction. The area of minimum swelling of gPMAA is observed at initial moment of time in the presence of only polyacid (ratio 6:0) due to the absence of additional dissociation of carboxyl groups in the result of equilibrium shift to the right (to proton formation). At the ratio 3:3 there is a formation of intramolecular crosslinks, which leads to destruction of formed during remote interaction intramolecular associates and as a result there is swelling decrease.

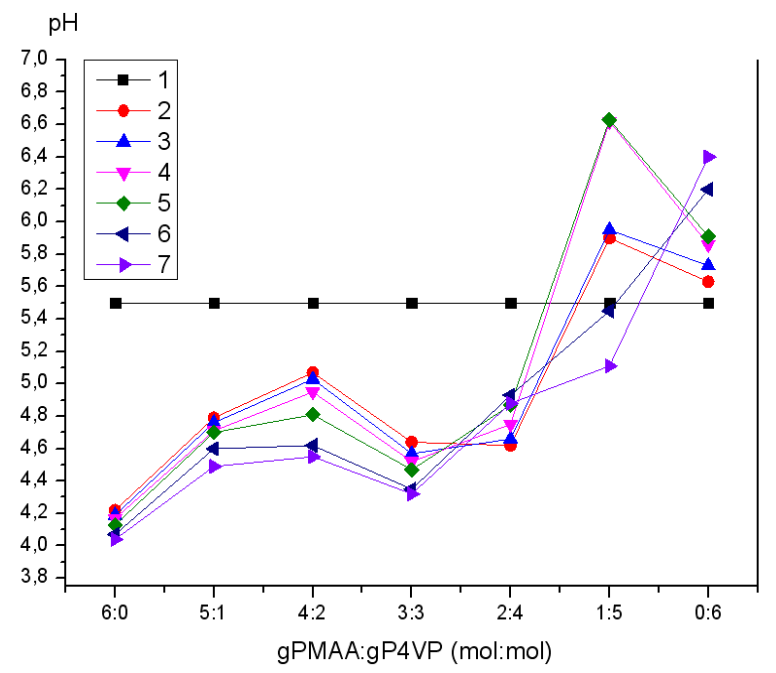

Fig. 2. Dependence of $\mathrm{pH}$ of water solutions on gPMAA and gP4VP hydrogels molar ratios in time (h): 0 (1); 0.5 (2); 1 (3); 2 (4); 6 (5); 24 (6) and 48 (7) 


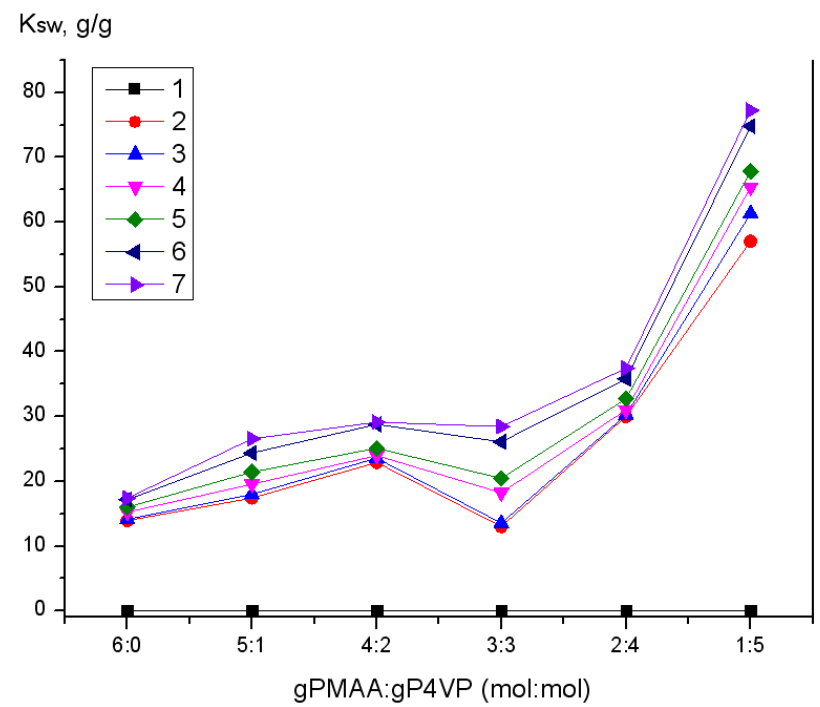

Fig. 3. Dependence of polymethacrylic acid swelling coefficient on gPMAA and gP4VP hydrogels molar ratios in time (h): 0 (1); $0.5(2) ; 1(3) ; 2$ (4); 6 (5); 24 (6) and 48 (7)

Dependence of poly-4-vinylpyridine hydrogel swelling coefficient on hydrogels molar ratios is presented in Fig. 4. Minimum swelling of polybasis occurs at hydrogels ratio 1:5 for the entire time of remote interaction of hydrogels in the intergel system. With polymethacrylic acid concentration increase there is an increase of swelling coefficient of polybasis. Clear maximum can be seen at gPMAA:gP4VP ratio 5:1 for $48 \mathrm{~h}$ of hydrogels remote interaction.

Comparing the values of electric conductivity, $\mathrm{pH}$ and swelling coefficients it is possible to conclude that in the result of mutual activation hydrogels are transferred into highly ionized state. Hydrogels maximum activation occurs at the ratio gPMAA:gP4VP=3:3. Hydrogel gP4VP is highly ionized at the ratio 5:1, while gPMAA hydrogel is highly ionized at predominance of polybasis (ratio 1:5).

\subsection{Study of Lanthanum Ions Sorption by Intergel System gPM AA-gP4V P}

From the results mentioned above it can be expected that phenomena of mutual activation of functional hydrogels should be also reflected in sorption processes of metals ions. To test this assumption sorption properties of the intergel system in relation to lanthanum ions were studied.

During lanthanum ions recovery by intergel system gPMAA-gP4VP there is also a change in electrochemical and conformational properties, though these changes are different from the situation when hydrogels interact in water medium.

Changes of specific electric conductivity of lanthanum nitrate solution in the presence of intergel

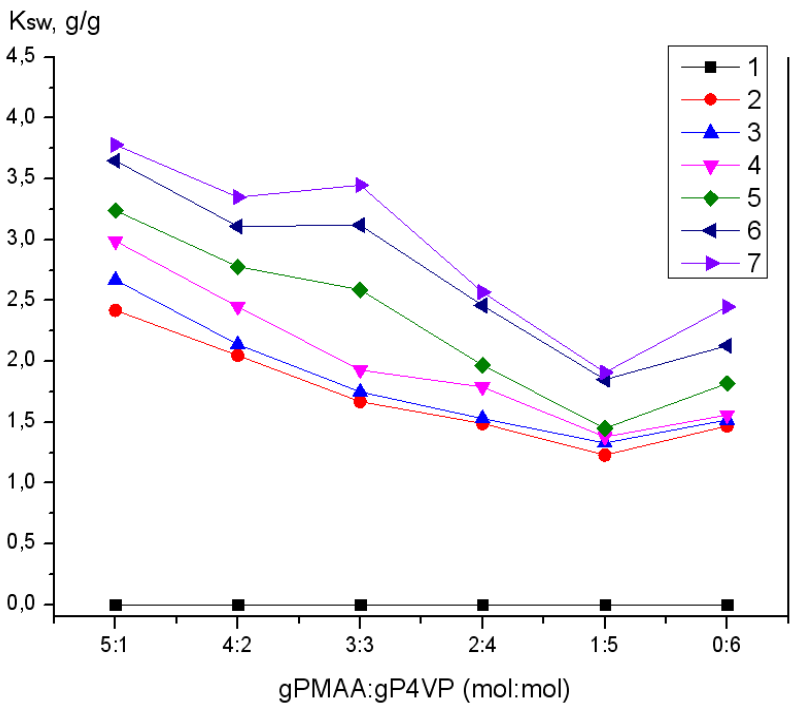

Fig. 4. Dependence of poly-4-vinylpyridine swelling coefficient on gPMAA and gP4VP hydrogels molar ratios in time (h): 0 (1); 0.5 (2); 1 (3); 2 (4); 6 (5); 24 (6) and 48 (7)

system gPMAA-gP4VP are presented in Fig. 5. From the obtained data it can be seen that during lanthanum ions sorption there are significant changes of conductivity. Electric conductivity decreases with time for all gPMAA:gP4VP ratios. Minimum conductivity of the system is reached at hydrogels ratio $3: 3$ for $48 \mathrm{~h}$ of remote interaction. It should be noted that low conductivity values are also observed at the ratios of gPMAA:gP4VP = $=5: 1$ and 1:5. In the solution in addition to present $-\mathrm{COO}^{-}$ and $\mathrm{H}^{+}$ions there are ions formed at three-stage lanthanum dissociation. The following chemical reactions occur:

1) Lanthanum nitrate dissociation in parallel with carboxyl groups dissociation;

2) Mutual activation of hydrogels due to proton binding by poly-4-vinylpyridine [14];

3) Sorption of lanthanum ions by hydrogels.

These reactions have impact on electrochemical equillibrium and depending on the dominance of each of them there will be changes of specific electric conductivity values.

In Fig. 6 dependence of hydrogen ions concentration on polymethacrylic acid and poly-4-vinylpyridine hydrogels molar ratios is shown. There is an increase of hydrogen ions concentration with time. Minimum values of $\mathrm{pH}$ are observed when there is only polyacid in solution (ratio 6:0) and at the ratio gPMAA:gP4VP $=5: 1$. This phenomenon is due to the fact that firstly there is hydrogels ionization due to mutual activation and after that ionization occurs due to formation of coordination bonds with lanthanum ions. 


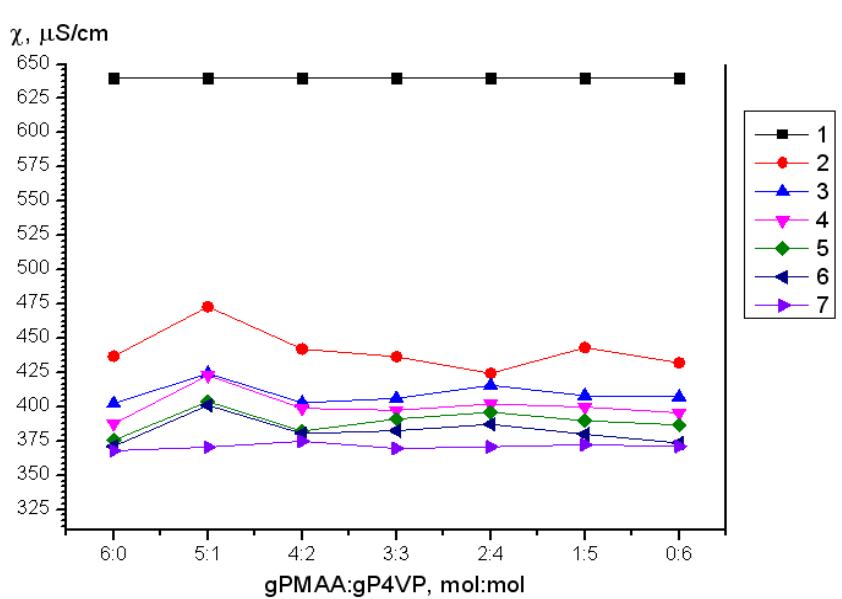

Fig. 5. Dependence of specific electric conductivity on gPMAA and gP4VP hydrogels molar ratios in time (h) in the medium of 6-water lanthanum nitrate: 0 (1); 0.5 (2); 1 (3); 2 (4); 6 (5); 24 (6) and 48 (7)

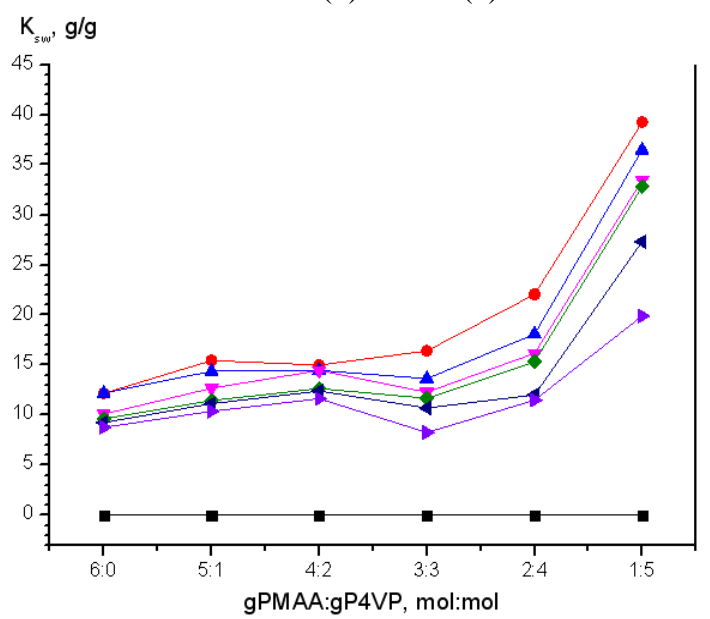

Fig. 7. Dependence of polymethacrylic acid swelling coefficient on gPMAA and gP4VP hydrogels molar ratios in time (h) in the medium of 6-water lanthanum nitrate: $0(1) ; 0.5$ (2); 1 (3); 2 (4); 6 (5); 24 (6) and 48 (7)

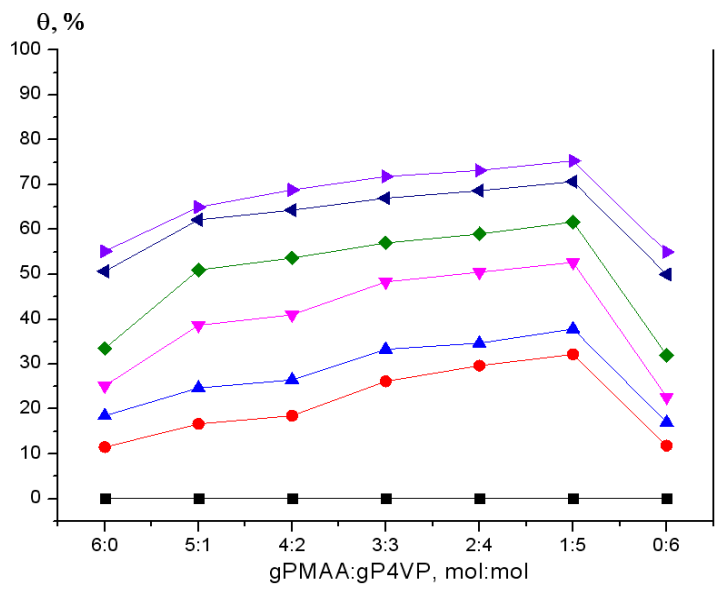

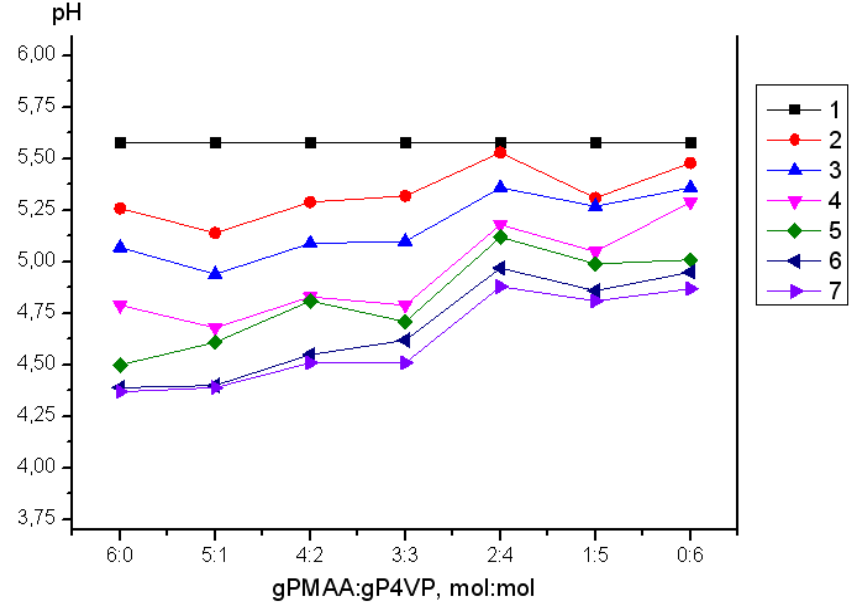

Fig. 6. Dependence of pH on gPMAA and gP4VP hydrogels molar ratios in time $(\mathrm{h})$ in the medium of 6 -water lanthanum nitrate: 0 (1); 0.5 (2); 1 (3); 2 (4); 6 (5); 24 (6) and 48 (7)

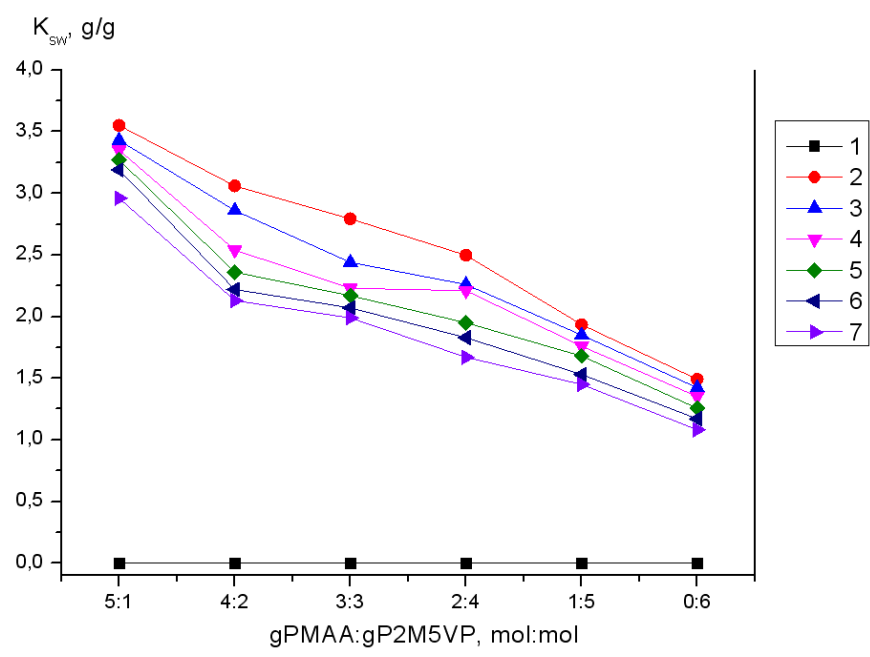

Fig. 8. Dependence of poly-4-vinylpyridine swelling coefficient on gPMAA and gP4VP hydrogels molar ratios in time (h) in the medium of 6-water lanthanum nitrate: 0 (1); 0.5 (2); 1 (3); 2 (4); 6 (5); 24 (6) and 48 (7)

Fig. 9. Dependence of polymer chain binding degree of lanthanum ions on gPMAA and gP4VP hydrogels molar ratios in time (h) in the medium of 6-water lanthanum nitrate: 0 (1); 0.5 (2); 1 (3); 2 (4); 6 (5); 24 (6) and 48 (7) 
Fig. 7 shows curves of dependence of gPMAA swelling coefficient during lanthanum ions sorption. Sharp increase of polymethacrylic acid hydrogel swelling occurs with increase of poly-4-vinylpyridine share in solution. Maximum values of swelling are observed at gPMAA:gP4VP $=1: 5$ ratio for $1 \mathrm{~h}$ of hydrogels remote interaction. After that there is stepwise decrease of swelling. Minimum values of swelling coefficient are observed at hydrogels ratio 5:1. There are no same charges on functional groups, which provides unfolding of macromolecule globe due to lanthanum ions sorption and, as a result, polymethacrylic acid hydrogel swelling decrease occur.

Dependence of swelling coefficient of basic hydrogel poly-4-vinylpyridine on gPMAA:gP4VP molar ratios is shown in Fig. 8. Change of swelling coefficient of basic hydrogel occurs similarly to swelling of polyacid. Firstly there is significant increase of swelling; maximum values of swelling coefficient being reached at gPMAA:gP4VP $=5: 1$ ratio for $1 \mathrm{~h}$ of hydrogels remote interaction. Subsequently, swelling decrease occurs like in case with polymethacrylic acid. Minimum swelling of poly-4-vinylpyridine is observed in the presence of only polybasis (ratio gPMAA:gP4VP=0:6) due to the absence of phenomenon of hydrogels mutual activation.

In Fig. 9 total binding degree of polymer chain is presented. As seen from the figure ratios when there is only polyacid or polybasis (gPMAA:gP4VP $=6: 0$ and 0:6) have lower binding degree in comparison with the ratios when there are 2 hydrogels in solution. Maximum polymer chain binding degree is observed at the ratio gPMAA:gP4VP $=1: 5$, it has the value of $73.13 \%$. This is due to the fact that hydrogels of polymethacrylic acid and poly-4-vinylpyridine are in highly ionized state due to hydrogels mutual activation in intergel systems.

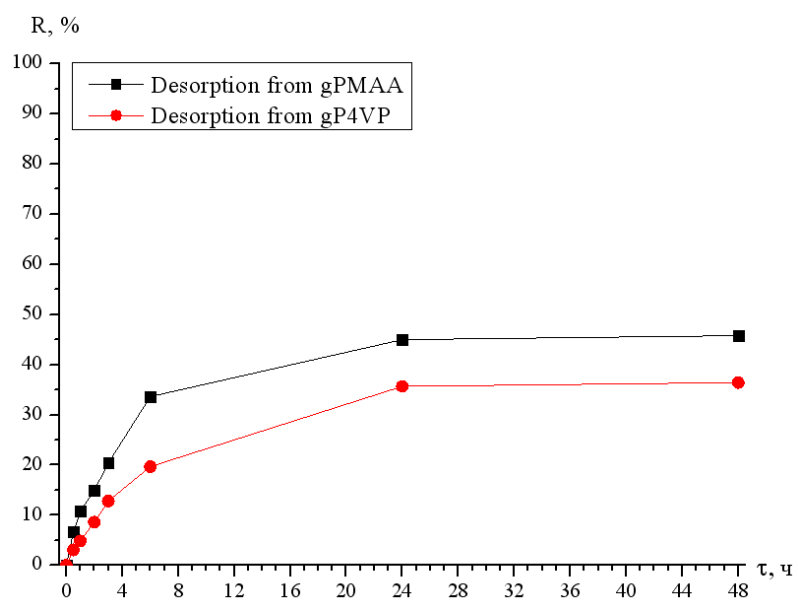

Fig. 10. Kinetics of lanthanum ions desorption by $96 \%$ ethyl alcohol from hydrogels matrix

\subsection{Comparison of Sorption Ability of Intergel System and Individual Hydrogels}

Lanthanum nitrate is present in solution in dissociated state. Dissociation of lanthanum nitrate occurs in 3 stages, in which constant of $1^{\text {st }}$ stage is much higher than constants of $2^{\text {nd }}$ and $3^{\text {rd }}$ stage. In this regard intergel system binds dissociated ions in accordance with different mechanisms. Nitrate of lanthanum formed in first stage is bound by ionic mechanism. Products of the second and third stage are bound due to coordination.

It should be noted that in intergel system gPMAAgP4VP hydrogels mutual activation leads to significant changes of their electrochemical and conformational properties. Also there is an increase in binding degree of polymer chain of each hydrogel in intergel pair. Binding degree of individual hydrogels gPMAA and gP4VP is 55.17 and $55.00 \%$, respectively. At the ratio gPMAA:gP4VP = 1:5 in intergel system gPMAA-gP4VP binding degree is $73.13 \%$. Increase of polymer chain binding degree is due to different conformational changes in the structure of polymer macromolecules (ex. unfolding of globe due to repulsion of same-charged ions of internode links of macromolecule) during hydrogels ionization.

\subsection{Features of Lanthanum Ions Desorption from Hydrogels Matrix}

For desorption of metal ions from polymer complexes strong mineral acids or polar organic solvents can be used [12].

Kinetics of lanthanum ions desorption by ethyl alcohol and nitric acid from individual hydrogels (ratio gPMAA:gP4VP = 1:5) are presented in Figs. 10 and 11.

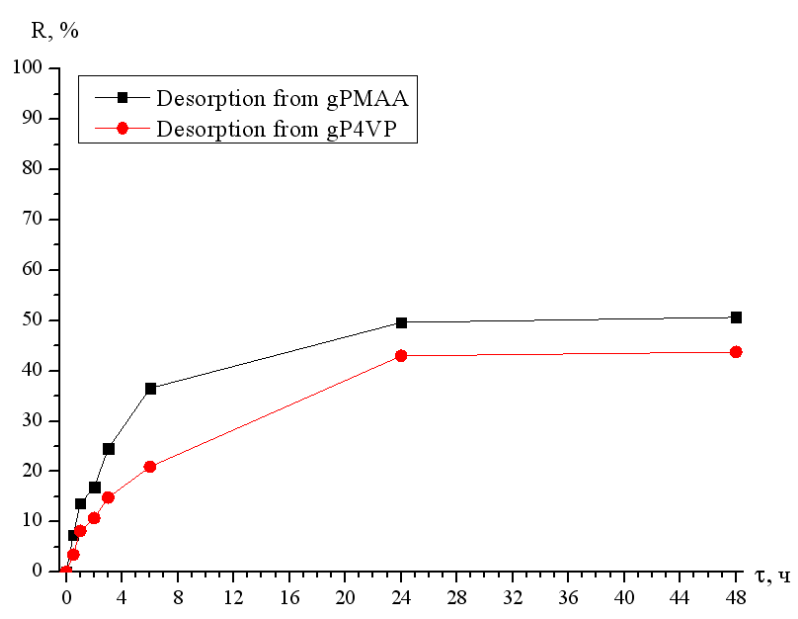

Fig. 11. Kinetics of lanthanum ions desorption by $2 \mathrm{M}$ nitric acid from hydrogels matrix 
Lanthanum ions desorption kinetics by ethyl alcohol is presented in Fig. 10. As seen from the figure sharp increase of lanthanum concentration is observed for $6 \mathrm{~h}$. After that there is further increase becoming insignificant after $24 \mathrm{~h}$. Total desorption degree after $24 \mathrm{~h}$ is $82.26 \%$.

Kinetics of lanthanum ions desorption by nitric acid as desorbent is shown in Fig. 11. Desorption process occurs similarly to desorption with ethyl alcohol - the highest desorption occurs during $6 \mathrm{~h}$. After that there is an increase of lanthanum ions concentration in desorbent, reaching final values for $48 \mathrm{~h}$. Desorption degree with nitric acid is $94.43 \%$.

\section{Conclusions}

The obtained results allow to make the following conclusions. Polymer macromolecules mutual activation leads to significant changes in electrochemical and conformational properties of hydrogels. Based on the data of conductivity, $\mathrm{pH}$ and swelling coefficient it can be concluded that lanthanum ions sorption by polymer hydrogels occurs. Maximum total binding degree of polymer chain $(73.13 \%)$ is observed at gPMAA:gP4VP ratio of 1:5. Desorption degree at lanthanum ions recovery from hydrogels matrix by ethyl alcohol is $82.26 \%$. Desorption degree by nitric acid is $94.43 \%$. Mutual activation by gPMAA and gP4VP hydrogels in intergel system provides higher sorption degree for intergel pair as compared to individual hydrogels.

\section{Acknowledgments}

This work was done with financial support of science committee of Ministry of Education and Science of Republic of Kazakhstan.

\section{References}

[1] Alimbekova B., Korganbayeva Zh., Himersen H. et al.: J. Chem. Chem. Eng., 2014, 3, 265.

[2] Jumadilov T., Kaldayeva S. et al.: $3^{\text {rd }}$ Int. Caucasian Symp. on Polymers and Advanced Materials, Georgia, Tbilisi 2013, 191.
[3] Jumadilov T.: Int. Conf. of Lithuanian Chemical Society "Chemistry and Chemical Technology", Lithuania, Kaunas 2014, 226.

[4] Alimbekova B., Jumadilov T., Korganbayeva Zh. et al.: Bul. d'EUROTALENT-FIDJIP, 2014, 5, 28.

[5] Erzhet B., Jumadilov T., Korganbayeva Zh. et al.: Bul. d'EUROTALENT-FIDJIP, 2014, 5, 41.

[6] Jumadilov T., Himersen H., Kaldayeva S. et al: J. Mat. Sci. Eng. B., 2014, 4, 209.

[7] Jumadilov T.: Ind. Kazakhstan, 2011, 2, 70.

[8] Bekturov E., Suleimenov I.: Polimernie Hidrogeli. Almaty 1998.

[9] Bekturov E., Jumadilov T.: Izv. Nats. Akad. Nauk Resp. Kazakhstan, Ser. Khim., 2010, 3, 86.

[10] Bekturov E., Jumadilov T., Korganbayeva Zh.: Vestnik Kaz. Nats. Univ., 2010, 3, 108.

[11] Jumadilov T., Shaltykova D., Suleimenov I.: AustrianSlovenian Polymer Meeting, Slovenia 2013, 51.

[12] Tereshenkova A., Statkus M., Tihomirova T. et al.: Vestnik Mosk. Univ., 2013, 4, 203.

[13] Petruhin O.: Praktikum po Fiziko-Khimicheskim Metodam Analiza. Academkniga, Moskva 1987.

[14] Jumadilov T., Abilov Zh., Kaldayeva S. et al.: J. Chem. Eng. Chem. Res., 2014, 4, 253.

Received: April 29, 2016 / Revised: J uly 13, 2016 / Accepted: J anuary 12, 2017

\section{ВЗАСМНА АКТИВАЦІЯ І СОРБЦІЯ РІДКО-ЗШИТОЇ СІТКИ В ІНТЕРГЕЛЕВІЙ СИСТЕМІ НА ОСНОВІ ГІДРОГЕЛЮ ПОЛІМЕТАКРИЛОВОЇ КИСЛОТИ ТА ПОЛІ-4-ВІНІЛПІРИДИНУ ВІДНОСНО ЛАНТАН ЙОНІВ}

Анотація. Досліджено сорбиійну здатність інтергелевої системи гідрогель поліметакрилової кислоти (gРМАA) полі-4-вінілпіридин (gP4VP) відносно йонів лантану. Вивчено взаємну активацію гідрогелів у водному середовищі, зокрема залежність коефіиієнта набухання, питомої електропровідності і рН водних розчинів. Визначено, що максимальна активачія гідрогелей відбувається за мольного співвідношення gPMAA:gP4VP = 3:3. Максимальна загальна ступінь зв'язування полімерного ланцюга в інтергелевій системі спостерігається при співвіднотенні дРМАA: gР4VP = 1: $5 \mathrm{ma}$ становить $73.13 \%$, що $є$ значно вищою, ніж для окремих гідрогелів: для gРМАA 55.17 \%, для gP4VP - $55.00 \%$. Показано, що при зазначеному співвідношенні значно змінюються величини електрохімічних, конформаційних $i$ сорбиійних ступенів вихідних макромолекул в інтергелевій системі.

Ключові слова: інтергелева система, поліметакрилова кислота, полі-4-вінілпіридин, дистаниійна взаємодія, йони La ${ }^{3+}$, сорбиія, десорбиія. 